\title{
Riemann Indefinite Integral of Functions of Real Variable ${ }^{1}$
}

\author{
Yasunari Shidama \\ Shinshu University \\ Nagano, Japan
}

\author{
Noboru Endou \\ Gifu National College of Technology \\ Japan
}

Summary. In this article we define the Riemann indefinite integral of functions of real variable and prove the linearity of that [1]. And we give some examples of the indefinite integral of some elementary functions. Furthermore, also the theorem about integral operation and uniform convergent sequence of functions is proved.

MML identifier: INTEGRA7, version: 7.8 .05 4.84.971

The papers [24], [25], [3], [23], [5], [13], [2], [26], [7], [21], [8], [10], [4], [17], [16], [15], [14], [19], [20], [6], [9], [11], [18], [12], [27], and [22] provide the terminology and notation for this paper.

\section{Preliminaries}

For simplicity, we adopt the following rules: $a, b, r$ are real numbers, $A$ is a non empty set, $X, x$ are sets, $f, g, F, G$ are partial functions from $\mathbb{R}$ to $\mathbb{R}$, and $n$ is an element of $\mathbb{N}$.

Next we state a number of propositions:

\footnotetext{
${ }^{1}$ This work has been partially supported by the MEXT grant Grant-in-Aid for Young Scientists (B)16700156.
} 
(1) Let $f, g$ be functions from $A$ into $\mathbb{R}$. Suppose $\operatorname{rng} f$ is upper bounded and $\operatorname{rng} g$ is upper bounded and for every set $x$ such that $x \in A$ holds $|f(x)-g(x)| \leq a$. Then sup rng $f-\sup \operatorname{rng} g \leq a$ and $\sup \operatorname{rng} g-\sup \operatorname{rng} f \leq$ $a$.

(2) Let $f, g$ be functions from $A$ into $\mathbb{R}$. Suppose $\operatorname{rng} f$ is lower bounded and $\operatorname{rng} g$ is lower bounded and for every set $x$ such that $x \in A$ holds $|f(x)-g(x)| \leq a$. Then inf $\operatorname{rng} f-\inf \operatorname{rng} g \leq a$ and $\inf \operatorname{rng} g-\inf \operatorname{rng} f \leq a$.

(3) If $f\lceil X$ is bounded on $X$, then $f$ is bounded on $X$.

(4) For every real number $x$ such that $x \in X$ and $f\lceil X$ is differentiable in $x$ holds $f$ is differentiable in $x$.

(5) If $f\lceil X$ is differentiable on $X$, then $f$ is differentiable on $X$.

(6) Suppose $f$ is differentiable on $X$ and $g$ is differentiable on $X$. Then $f+g$ is differentiable on $X$ and $f-g$ is differentiable on $X$ and $f g$ is differentiable on $X$.

(7) If $f$ is differentiable on $X$, then $r f$ is differentiable on $X$.

(8) Suppose for every set $x$ such that $x \in X$ holds $g(x) \neq 0$ and $f$ is differentiable on $X$ and $g$ is differentiable on $X$. Then $\frac{f}{g}$ is differentiable on $X$.

(9) If for every set $x$ such that $x \in X$ holds $f(x) \neq 0$ and $f$ is differentiable on $X$, then $\frac{1}{f}$ is differentiable on $X$.

(10) Suppose $a \leq b$ and $\left[{ }^{\prime} a, b^{\prime}\right] \subseteq X$ and $F$ is differentiable on $X$ and $F_{\lceil X}^{\prime}$ is integrable on $\left[{ }^{\prime} a, b^{\prime}\right]$ and $F_{\mid X}^{\prime}$ is bounded on $\left[{ }^{a} a, b^{\prime}\right]$. Then $F(b)=$ $\int_{a}^{b}\left(F_{\lceil X}^{\prime}\right)(x) d x+F(a)$.

\section{The Definition of Indefinite Integral}

Let $X$ be a set and let $f$ be a partial function from $\mathbb{R}$ to $\mathbb{R}$. The functor IntegralFuncs $(f, X)$ yields a set and is defined by the condition (Def. 1).

(Def. 1) $\quad x \in \operatorname{IntegralFuncs}(f, X)$ if and only if there exists a partial function $F$ from $\mathbb{R}$ to $\mathbb{R}$ such that $x=F$ and $F$ is differentiable on $X$ and $F_{\lceil X}^{\prime}=f\lceil X$.

Let $X$ be a set and let $F, f$ be partial functions from $\mathbb{R}$ to $\mathbb{R}$. We say that $F$ is an integral of $f$ on $X$ if and only if:

(Def. 2) $F \in \operatorname{IntegralFuncs}(f, X)$.

The following propositions are true:

(11) If $F$ is an integral of $f$ on $X$, then $X \subseteq \operatorname{dom} F$.

(12) Suppose $F$ is an integral of $f$ on $X$ and $G$ is an integral of $g$ on $X$. Then $F+G$ is an integral of $f+g$ on $X$ and $F-G$ is an integral of $f-g$ on $X$. 
(13) If $F$ is an integral of $f$ on $X$, then $r F$ is an integral of $r f$ on $X$.

(14) If $F$ is an integral of $f$ on $X$ and $G$ is an integral of $g$ on $X$, then $F G$ is an integral of $f G+F g$ on $X$.

(15) Suppose for every set $x$ such that $x \in X$ holds $G(x) \neq 0$ and $F$ is an integral of $f$ on $X$ and $G$ is an integral of $g$ on $X$. Then $\frac{F}{G}$ is an integral of $\frac{f G-F g}{G G}$ on $X$.

(16) Suppose that

(i) $a \leq b$,

(ii) $\left[{ }^{\prime} a, b^{\prime}\right] \subseteq \operatorname{dom} f$,

(iii) $f$ is continuous on $\left[{ }^{\prime} a, b^{\prime}\right]$,

(iv) $] a, b[\subseteq \operatorname{dom} F$, and

(v) for every real number $x$ such that $x \in] a, b\left[\right.$ holds $F(x)=\int_{a}^{x} f(x) d x+$ $F(a)$.

Then $F$ is an integral of $f$ on $] a, b[$.

(17) Let $x, x_{0}$ be real numbers. Suppose $f$ is continuous on $[a, b]$ and $\left.x \in\right] a, b[$ and $\left.x_{0} \in\right] a, b[$ and $F$ is an integral of $f$ on $] a, b\left[\right.$. Then $F(x)=\int_{x_{0}}^{x} f(x) d x+$ $F\left(x_{0}\right)$.

(18) Suppose $a \leq b$ and $\left[{ }^{\prime} a, b^{\prime}\right] \subseteq X$ and $F$ is an integral of $f$ on $X$ and $f$ is integrable on $\left[{ }^{\prime} a, b^{\prime}\right]$ and $f$ is bounded on $\left[{ }^{\prime} a, b^{\prime}\right]$. Then $F(b)=\int_{a}^{b} f(x) d x+$ $F(a)$.

(19) Suppose $a \leq b$ and $[a, b] \subseteq X$ and $f$ is continuous on $X$. Then $f$ is continuous on $\left[{ }^{\prime} a, b^{\prime}\right]$ and $f$ is integrable on $\left[{ }^{\prime} a, b^{\prime}\right]$ and $f$ is bounded on $\left[{ }^{\prime} a, b^{\prime}\right]$.

(20) If $a \leq b$ and $[a, b] \subseteq X$ and $f$ is continuous on $X$ and $F$ is an integral of $f$ on $X$, then $F(b)=\int_{a}^{b} f(x) d x+F(a)$.

(21) Suppose that $b \leq a$ and $\left[{ }^{\prime} b, a^{\prime}\right] \subseteq X$ and $f$ is integrable on $\left[{ }^{\prime} b, a^{\prime}\right]$ and $g$ is integrable on $\left[{ }^{\prime} b, a^{\prime}\right]$ and $f$ is bounded on $\left[{ }^{\prime} b, a^{\prime}\right]$ and $g$ is bounded on $\left[{ }^{\prime} b, a^{\prime}\right]$ and $X \subseteq \operatorname{dom} f$ and $X \subseteq \operatorname{dom} g$ and $F$ is an integral of $f$ on $X$ and $G$ is an integral of $g$ on $X$. Then $F(a) \cdot G(a)-F(b) \cdot G(b)=$ $\int_{b}^{a}(f G)(x) d x+\int_{b}^{a}(F g)(x) d x$.

(22) Suppose that $b \leq a$ and $[b, a] \subseteq X$ and $X \subseteq \operatorname{dom} f$ and $X \subseteq \operatorname{dom} g$ and 
$f$ is continuous on $X$ and $g$ is continuous on $X$ and $F$ is an integral of $f$ on $X$ and $G$ is an integral of $g$ on $X$. Then $F(a) \cdot G(a)-F(b) \cdot G(b)=$ $\int_{b}^{a}(f G)(x) d x+\int_{b}^{a}(F g)(x) d x$.

\section{Examples of Indefinite Integral}

We now state several propositions:

(23) The function sin is an integral of the function cos on $\mathbb{R}$.

(24) (The function $\sin )(b)-($ the function $\sin )(a)=\int_{a}^{b}($ the function $\cos )(x) d x$.

(25) (-1) (the function cos) is an integral of the function $\sin$ on $\mathbb{R}$.

(26) (The function $\cos )(a)-($ the function $\cos )(b)=\int_{a}^{b}($ the function $\sin )(x) d x$.

(27) The function exp is an integral of the function exp on $\mathbb{R}$.

(28) (The function exp) $(b)-($ the function $\exp )(a)=\int_{a}^{b}($ the function $\exp )(x) d x$.

(29) ${ }_{\mathbb{Z}}^{n+1}$ is an integral of $(n+1)_{\mathbb{Z}}^{n}$ on $\mathbb{R}$.

(30) $\quad\left({ }_{\mathbb{Z}}^{n+1}\right)(b)-\left({ }_{\mathbb{Z}}^{n+1}\right)(a)=\int_{a}^{b}\left((n+1)_{\mathbb{Z}}^{n}\right)(x) d x$.

\section{Uniform Convergent Functional Sequence}

We now state the proposition

(31) Let $H$ be a sequence of partial functions from $\mathbb{R}$ into $\mathbb{R}$ and $r_{1}$ be a sequence of real numbers. Suppose that

(i) $a<b$,

(ii) for every element $n$ of $\mathbb{N}$ holds $H(n)$ is integrable on ['a, $\left.b^{\prime}\right]$ and $H(n)$ is bounded on [' $\left.a, b^{\prime}\right]$ and $r_{1}(n)=\int_{a}^{b} H(n)(x) d x$, and

(iii) $\quad H$ is uniform-convergent on $\left[{ }^{\prime} a, b^{\prime}\right]$.

Then $\lim _{\left[{ }^{\prime} a, b^{\prime}\right]} H$ is bounded on $\left[{ }^{\prime} a, b^{\prime}\right]$ and $\lim _{\left[{ }^{\prime} a, b^{\prime}\right]} H$ is integrable on $\left[{ }^{\prime} a, b^{\prime}\right]$ and $r_{1}$ is convergent and $\lim r_{1}=\int_{a}^{b} \lim _{\left[^{\prime} a, b^{\prime}\right]} H(x) d x$. 


\section{REFERENCES}

[1] Tom M. Apostol. Mathematical Analysis. Addison-Wesley, 1969.

[2] Grzegorz Bancerek. The fundamental properties of natural numbers. Formalized Mathematics, 1(1):41-46, 1990.

[3] Grzegorz Bancerek. The ordinal numbers. Formalized Mathematics, 1(1):91-96, 1990.

[4] Grzegorz Bancerek and Krzysztof Hryniewiecki. Segments of natural numbers and finite sequences. Formalized Mathematics, 1(1):107-114, 1990.

[5] Czesław Byliński. The complex numbers. Formalized Mathematics, 1(3):507-513, 1990.

[6] Czesław Byliński. Finite sequences and tuples of elements of a non-empty sets. Formalized Mathematics, 1(3):529-536, 1990.

[7] Czesław Byliński. Functions from a set to a set. Formalized Mathematics, 1(1):153-164, 1990.

[8] Czesław Byliński. Partial functions. Formalized Mathematics, 1(2):357-367, 1990.

[9] Czesław Byliński. The sum and product of finite sequences of real numbers. Formalized Mathematics, 1(4):661-668, 1990.

[10] Czesław Byliński and Piotr Rudnicki. Bounding boxes for compact sets in $\mathcal{E}^{2}$. Formalized Mathematics, 6(3):427-440, 1997.

[11] Noboru Endou and Artur Korniłowicz. The definition of the Riemann definite integral and some related lemmas. Formalized Mathematics, 8(1):93-102, 1999.

[12] Noboru Endou, Katsumi Wasaki, and Yasunari Shidama. Definition of integrability for partial functions from $\mathbb{R}$ to $\mathbb{R}$ and integrability for continuous functions. Formalized Mathematics, 9(2):281-284, 2001.

[13] Krzysztof Hryniewiecki. Basic properties of real numbers. Formalized Mathematics, 1(1):35-40, 1990.

[14] Jarosław Kotowicz. Convergent real sequences. Upper and lower bound of sets of real numbers. Formalized Mathematics, 1(3):477-481, 1990.

[15] Jarosław Kotowicz. Convergent sequences and the limit of sequences. Formalized Mathematics, 1(2):273-275, 1990.

[16] Jarosław Kotowicz. Partial functions from a domain to the set of real numbers. Formalized Mathematics, 1(4):703-709, 1990.

[17] Jarosław Kotowicz. Real sequences and basic operations on them. Formalized Mathematics, 1(2):269-272, 1990.

[18] Beata Perkowska. Functional sequence from a domain to a domain. Formalized Mathematics, 3(1):17-21, 1992.

[19] Konrad Raczkowski and Paweł Sadowski. Real function continuity. Formalized Mathematics, 1(4):787-791, 1990.

[20] Konrad Raczkowski and Paweł Sadowski. Real function differentiability. Formalized Mathematics, 1(4):797-801, 1990.

[21] Konrad Raczkowski and Paweł Sadowski. Topological properties of subsets in real numbers. Formalized Mathematics, 1(4):777-780, 1990.

[22] Yasunari Shidama. The Taylor expansions. Formalized Mathematics, 12(2):195-200, 2004.

[23] Andrzej Trybulec. Subsets of complex numbers. To appear in Formalized Mathematics.

[24] Andrzej Trybulec. Tarski Grothendieck set theory. Formalized Mathematics, 1(1):9-11, 1990

[25] Zinaida Trybulec. Properties of subsets. Formalized Mathematics, 1(1):67-71, 1990.

[26] Edmund Woronowicz. Relations defined on sets. Formalized Mathematics, 1(1):181-186, 1990.

[27] Yuguang Yang and Yasunari Shidama. Trigonometric functions and existence of circle ratio. Formalized Mathematics, 7(2):255-263, 1998.

Received June 6, 2007 